\title{
Toward Interplanetary Space Weather: Strategies for Manned Missions to Mars
}

\author{
Claire Foullon, Norma Crosby, and Daniel Heynderickx
}

\section{July 2005}

By 2025 an international human mission to Mars may be a reality, with the Moon as a likely intermediate step. The desire to explore and send humans into interplanetary space is currently confronted with many uncertainties for assuring the crew's safety from radiation. One option to reduce these uncertainties and mitigate the potential radiation-induced health risks is to consider operational approaches. Future interplanetary manned missions will require global monitoring and warning systems capable of providing space weather forecasts and alerts and assuring adequate warning and protection from solar proton events (SPEs) during extravehicular activities. Aurora is the European Programme for the Exploration of the Solar System and will involve a program of robotic and manned missions. Within Aurora, the European Space Agency recently initiated a study entitled "Radiation Exposure and Mission Strategies for Interplanetary Manned Missions." Here we present part of the results from the work, a study on "Radiation Hazard and Space Weather Warning System." We review the strategies to adopt for the future development of interplanetary space weather.

\section{Radiation Hazard in Interplanetary Space}

In the late 1980s, low Earth orbit missions (e.g., the International Space Station, the HERMES space plane, etc.) were the main impetus for human space travel. A little over a decade later, space travel initiatives have reached farther into interplanetary space, and future missions to Mars are now under consideration.

Along with the continuous galactic cosmic ray (GCR) background present in space, SPEs are the main hazard for interplanetary missions due to their high energies $(>10 \mathrm{MeV})$ and propagation speeds (the highest-energy particles move with speeds close to the speed of light). For manned space flights, the largest concerns are SPEs with 70- to 100-MeV energies, capable of penetrating a space suit or vehicle skin and affecting the blood-forming organs, according to Wilson et al. in a 1999 report published in Radiation Measurements.

SPEs tend to be associated either with solar flare activity in complex sunspot groups or with massive, fast coronal mass ejections (CMEs). Solar flares are enormous energy releases of up to 1025 â€"1027 joules in the solar atmosphere, involving sudden bursts of particle acceleration, plasma heating, and bulk plasma motion. CMEs are huge plasma bubbles, threaded with magnetic field lines and ejected from the Sun over the course of several hours; they are seen as bright features moving outward through the corona at speeds up to $2000 \mathrm{~km} / \mathrm{s}$. The majority of SPEs are associated with CME-driven shocks, which take a day or two to reach Earth. In particular, large SPEs are associated with fast CMEs, often those whose Sun-Earth transit time is 1 day or less.

Because very large SPEs are an operational constraint on manned missions, it is necessary to develop methods for crew protection and forecast these potentially hazardous events. Knowledge of the radiation environment provides complementary operational approaches, such as the selection of a favorable launch time and duration within the solar cycle and the landing site on the Mars surface (or taking advantage of geological storm shelters on the Moon). During solar maximum, for example, the increase in the interplanetary magnetic field strength provides enhanced shielding of the heliosphere against penetrating GCR particles; the GCR population is therefore most intense during solar minimum. The GCR environment is well studied, and adequate models for its composition, spectral characteristics, and solar cycle dependence are available. Consequently, GCRs are a significant factor for multiyear space missions, but they should not have priority in an interplanetary radiation hazard warning system. 
In August 1972, between NASA's Apollo 16 and 17 manned space missions, one of the largest solar proton events ever recorded arrived at Earth. Published computer simulations of the radiation levels an astronaut inside a spacecraft would have experienced during this event found that the astronaut would have absorbed lethal doses of radiation within 10 hours after the start of the event. However, a number of studies concluded that a properly shielded astronaut would not have been exposed to radiation levels above the monthly recommended limit. In the future, with sufficient spacecraft shielding, SPEs will not stop us from going to Mars, but having a reliable SPE prediction capability will have high priority in order to minimize their impact on future interplanetary manned missions.

\section{Interplanetary Space Weather Development Strategies}

Terrestrial space weather systems rely on early warning from the L1 point between the Earth and the Sun, where the detection of SPEs provides a warning time of less than 1 hour. These systems can be used for Moon missions by relaying the L1 alert to the spacecraft's onboard warning system. This approach, however, is not feasible for true interplanetary missions away from the Sun-Earth line. The Mars-Sun-Earth angle is an important parameter for an Earth-based space weather system. A contributing factor to the occurrence of a SPE is the physical location of the active region on the solar surface relative to the observer and the characteristics of energy release in an accompanying solar flare. With backsided CMEs, for example, large errors can be made when locating their solar origin, estimating the onset time without detection of an associated flare, and estimating the shock velocity (without a kilometric type-II report). Consequently, for more than half of the mission duration, when Mars and Earth are on opposite sides of the Sun, the accuracy of predictions is compromised.

To ensure adequate warnings of potentially large SPE fluxes, the forecasting strategy is to provide continuous observations of the solar regions generating solar disturbances and to provide a global picture of the interplanetary medium, where solar wind disturbances propagate. Multispacecraft systems require a separation angle between the spacecrafts that allows stereoscopic imaging and reconstructions of spatial characteristics in the solar wind. These systemsâ€"and occasional monitorsâ€"may combine several individual spacecraft missions observing at the same time. They include not only interplanetary solar satellites but also Earth-based platforms.

In the case of a manned mission to Mars, one can assess the capability of future multispacecraft missions to ensure sufficient interplanetary space data coverage and forecasts. For instance, an examination of the relative positions of Mars and the Solar Terrestrial Relations Observatory (STEREO) twin spacecrafts shows that the STEREO mission will provide an occasional upstream solar wind and imaging monitor for any ongoing Mars mission. STEREO launch is planned for early 2006, near a time when Mars is closest to Earth (the closest approach will occur on 30 October 2005, and opposition will occur on 7 November 2005). In the ecliptic plane, the two STEREO spacecrafts (Ahead and Behind) will move at an angular rate of $22 \mathrm{deg} / \mathrm{yr}$ with respect to a fixed Earth-Sun line. One can then distinguish four chronological periods, according to the relative positions of Mars, the Earth, and the STEREO spacecrafts with respect to the Sun, as illustrated in Figure 1. In the period in Figure 1d, a Mars mission will benefit from the full capacity of the forecasting allowed by the combination of STEREO and the Sun-Earth line information. In the periods in Figures 1a and 1c, the coronagraph and EUV imaging from the trailing and leading STEREO spacecraft, respectively, will improve the solar activity monitoring of the limb region. Such data could allow mission scientists to anticipate the arrival of corotating structures at Mars and warn of potential SPEs. How ever, the quality of forecasting is difficult to assess at this time. In the period in Figure 1b, if an event is detected at the leading spacecraft and the associated active region at the Sun seems long-lived, improved corotation projections could warn of potential events at Mars, but with greater uncertainty.
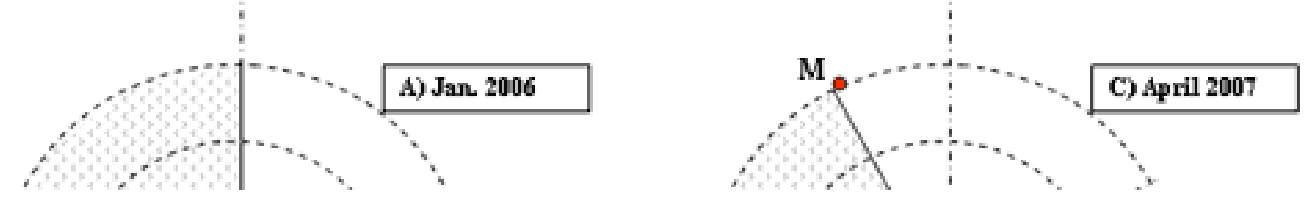


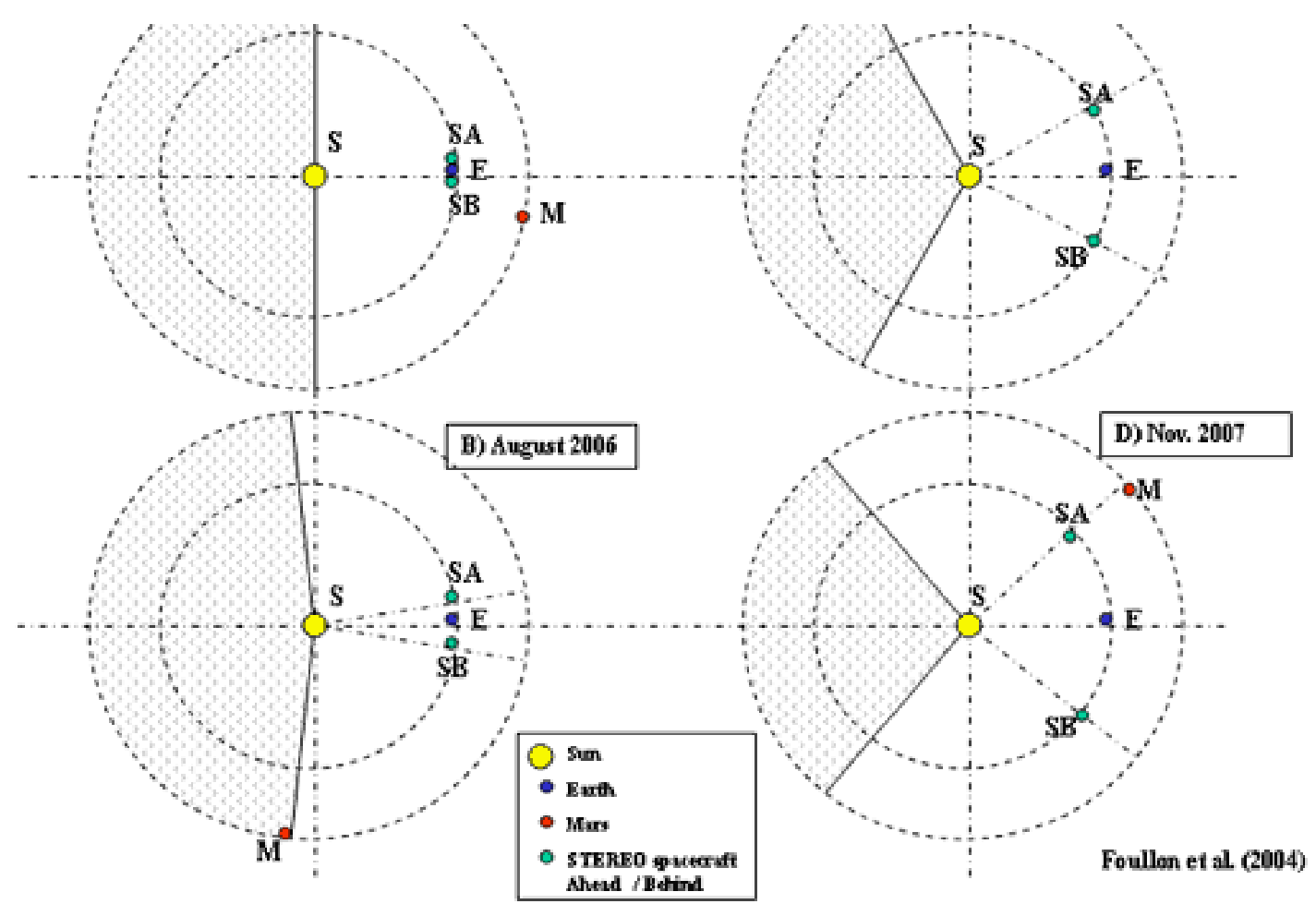

Figure 1. Ecliptic plane projection showing the relative positions of M ars and the STEREO-Earth L1 system for four different epochs: (a) STEREO lunar escapes, $\mathrm{M}$ ars is within $90 \hat{\mathrm{A}}^{\circ}$ of heliolongit ude behind the trailing STEREO spacecraft; (b) M ars enters the far side from the STEREO system for about 8 months; (c) Mars is within $90 \hat{\mathrm{A}}^{\circ}$ of heliolongitude ahead of the leading STEREO spacecraft; (d) Mars enters the zone covered by the STEREO-Earth L1 system. Sun, Earth, Mars and the STEREO spacecrafts Ahead and Behind are indicated by S, E, M, SA, and SB, respectively.

As long as the manned spacecraft or station is covered by the STEREO system, the forecasting operations would be feasible, but additional viewpoints are required beyond that coverage. Complementary solutions such as a sentinel on the far side of a STEREO system (similar in concept to the Far Side Sentinel) or a monitoring platform at the Sun-Mars LI point should be considered. The Earth-Mars distance, which varies approximately between 56 and 400 million $\mathrm{km}$, is an important parameter for telecommunications from Earth. Depending on the distance, the time required for radio communication from Earth to Mars varies between 3.1 and $22.2 \mathrm{~min}$. It is important to mention that when Mars is at conjunction, communication is not possible at all. For instance, during August 2002 , at the time of conjunction, published reports indicated that no data from the MARIE instrument onboard NASA's 2001 Mars Odyssey Orbiter were received for approximately 2 weeks. Using satellites orbiting the Sun-Mars L1 and L2 points as Earth-Mars communication relays could be an advantage, not only to avoid the loss of contact with Earth in the period of Earth-Mars conjunction, but also to use the satellite at $\mathrm{L} 1$ as a solar activity monitoring platform.

Essentially, four solar proton event models are available to spacecraft engineers for predicting long-term solar proton fluences: the King model, the Jet Propulsion Laboratory model, the emission of solar protons (ESP) total fluence and worst-case-event models, and the Moscow State University (MSU) fluence and peak flux model. The first three models are reviewed and made available for use on the Space Environment Information System (SPENVIS) Web site for registered users; the MSU model is available online at MSU, and will soon be incorporated into SPENVIS (see Table 1 for Web site links). Presently, the models rely on data collected at $1 \mathrm{AU}$. The usual method for estimating the energetic proton environment for a Mars mission is to take the solar proton observations at 1 AU and then extrapolate these observations to other radial distances (1.4â€"1.6 AU). However, the radial gradient associated with the shock acceleration is not well understood. Smart and Shea suggest in a 2003 Advances in Space Research article that the radial extrapolations expected by a power law geometry only apply to specific types of well-connected solar flare-associated events, and that they do not apply to the case of general shock accelerated events. Therefore the method of radial extrapolation is presently not reliable. In order to improve the solar proton fluence models on Mars and give more reliable estimates for mission planning, models need to be updated with data collected by missions to Mars and interplanetary space. Also, no event prediction is obtained by using the current models, only total mission fluence. 


\begin{tabular}{|c|c|}
\hline \multicolumn{2}{|l|}{ a-1) Interplanetary solar satellites } \\
\hline STEREO (2005) & 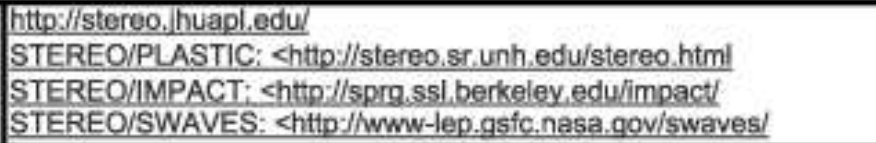 \\
\hline Solar Sentinels (2008-2009) & hitto///ws. asic.nasa gov/docs/MOWG06 03/Sentinels.pdi \\
\hline RSA InterHelios (2007-2008) & hitto/hwww izmiran. rssi.ru/space/solar/ihelios htm \\
\hline ISAS L5 mission (2008-2013) & hitto:/hilws.gsic.nasa.govicr kickofi.pdf \\
\hline ESA Solar Orbiter (2013) & hitto://sci.esa. int/science-e/www/area/index.cfm?tareaid=45 \\
\hline Solar Probe (2012) & htto://solarprobe.gsfc.nasa.gov/ \\
\hline \multicolumn{2}{|l|}{ a-2) Earth based platforms } \\
\hline SOHO & hitto:/sohowww nascom.nasa.gov \\
\hline SOLAR-B (2006) & hittp:I/science.nasa.gov/ssvpad/solar/solar-b.stm \\
\hline SDO (2007) & hitto://sdo.gstc.nasa.gov! \\
\hline \multicolumn{2}{|l|}{ a-3) Oecasional monitors } \\
\hline CASSINI RPWS & 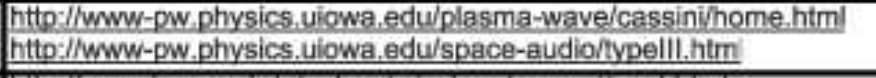 \\
\hline ISAS Nozomi & 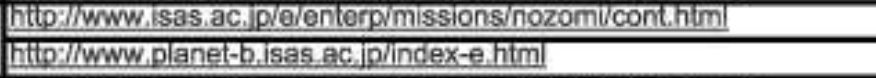 \\
\hline BepiColombo Project & hitp://sci.esa.int/scence-e/www/area/index.cim?tareaid=30 \\
\hline ESA Rosetta & hito:/hww.esa intexport/SPECIALS/Rosettalindex.htm \\
\hline \multicolumn{2}{|l|}{ b) Martian radiation environment } \\
\hline Phobos-2 & $\begin{array}{l}\text { hittp://nssdc.gs/c.nasa.gov/space/space phys/phobos2.himl } \\
\text { http://heasarc. gsfc.nasa.gov/docs/heasarc/missionsiphobos } 2 . \mathrm{htm}\end{array}$ \\
\hline MARIE/NASA 2001 Mars Odyssey & hitto://mars.jpLnasa.gov/odyssey/lechnology/marie.hitm \\
\hline ESA Mars Express & hitto://sci.esa.indscience-e/www/area/index.cfm?fareaid =9 \\
\hline ASPERA-3 & 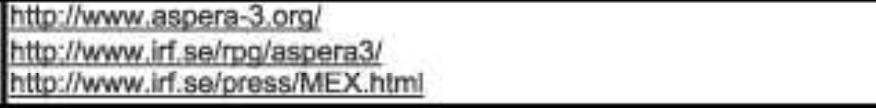 \\
\hline \multicolumn{2}{|l|}{ c) Miscellaneous } \\
\hline ESA/Aurora & hitto:/hwww.esa.int/export/SPECCIALS/Auroral \\
\hline ILWS & 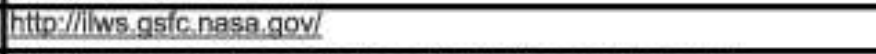 \\
\hline $\begin{array}{l}\text { SPENVIS solar proton fluence models } \\
\text { (for registered users) }\end{array}$ & $\begin{array}{l}\text { hittp://www.spenvis.oma.be/spenvis/help/models/sep,html } \\
\text { hittp://www, spenvis.oma.be/spenvis/help/background/flare/flare hitml }\end{array}$ \\
\hline MSU fluence and peak flux model & hittp://srd.sinp.msu.ru/models/sep2004.html \\
\hline
\end{tabular}

Table 1. Web sites on (a) missions of interest for multispacecraft systems (instrumentation, data, etc.), (b) past and current missions of particular interest for dat a collected about the Martian environment, and (c) miscellaneous programs and models.

Summing up, the Mars orbital parameters presented in turn in this review (the Earth-Sun-Mars angle, the Mars-Earth distance, and the Sun-Mars distance) tell us what strategies to adopt for the future development of interplanetary space weather forecasting. These strategies can be summarized into three requirements: having real-time observations from simultaneous missions at complementing positions and orbits, with appropriate and complementary instruments; improving the reliability of onboard warning and forecasting systems; and enhancing the development of interplanetary models to be tested against data. The past, present, and future missions into interplanetary space open the challenging opportunity to reach a comprehensive understanding of the processes at work in $\mathrm{CME}$ initiation, shock propagation, and particle acceleration. One way forward is to create working groups, such as those suggested to accomplish International Living With a Star (ILWS) goals. The potential of having an international fleet of spacecraft (e.g., Solar Orbiter, BepiColombo-MMO, the Solar Sentinels, Solar Probe, the Japanese L5 mission, and the Russian InterHelios spacecraft) and investigating the synergism of complementary observations may lead to combined and alternative mission scenarios.

Our ability to protect the crew of interplanetary missions from SPEs depends on the future of these missions and the know-how they will bring. With the associated development of real-time models and forecasts, it will be possible to decide on the choice of multispacecraft missions and minimum instrument packages necessary to assure continuous monitoring of the Sun and outer space. In general, we may safely say that aside from traditional in situ instruments (solar wind analyzer, magnetometer, and energetic particle detector), remote sensing instrumentsâ€"including a full disk imager, a Doppler magnetograph, and a coronagraphâ€"are essential as well. In particular, we need to make sure that future Earth-based platforms provide all nec essary 
instruments, not only for terrestrial space weather and missions to the Moon, but also for the development of our interplanetary space weather expertise.

\section{Acknowle dgments}

This work was performed under ESTEC contract 17696/03/NL/AG, Radiation Exposure and Mission Strategies for Interplanetary Manned Missions.

\section{References}

Foullon, C., A. Holmes-Siedle, N. B. Crosby, and D. Heynderickx (2004), Radiation Hazard and Space Weather Warning System, work-package, in Radiation Exposure and Mission Strategies for Interplanetary Manned Missions (REMSIM), Eur. Space Res. and Technol. Cent., Noordwijk, Netherlands.

C. Foullon is a researcher in the Department of Physics, University of Warwick, Coventry, UK.

N. B. Crosby and D. Heynderickx are researchers at the Belgian Institute for Space Aeronomy, Brussels, Belgium.

Citation: Foullon, C., N. Crosby, and D. Heynderickx (2005), Toward Interplanetary Space Weather: Strategies for Manned M issions to Mars, Space Weather, 3, S07004, doi:10.1029/2004SW000134.

Copyright 2005 by the American Geophysical Union. 